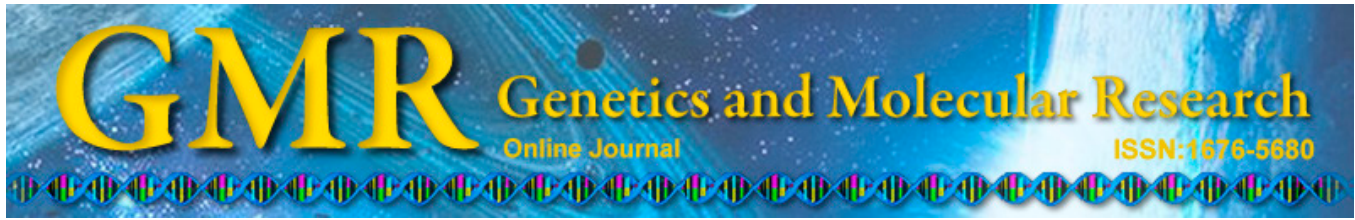

\title{
Effects of musk ketone on nerve recovery after spinal cord injury
}

\author{
L. Guo ${ }^{1}$, Z.X. Quan' ${ }^{2}$ Z.H. Zhao' ${ }^{2}$ K. Tang ${ }^{2}$, Y.S. Ou ${ }^{2}$ and D.M. Jiang ${ }^{2}$ \\ ${ }^{1}$ Department of Orthopedics, \\ University-Town Hospital of Chongqing Medical University, Chongqing, China \\ ${ }^{2}$ Department of Orthopedics, \\ The First Affiliated Hospital of Chongqing Medical University, Chongqing, China \\ Corresponding author: D.M. Jiang \\ E-mail: jdmmjd@yeah.net / jdm571026@vip.163.com
}

Genet. Mol. Res. 14 (2): 2958-2963 (2015)

Received March 25, 2014

Accepted October 7, 2014

Published April 10, 2015

DOI http://dx.doi.org/10.4238/2015.April.10.4

\begin{abstract}
The present study aimed to determine the effects of musk ketone on nerve recovery in rats after spinal cord injury. A total of $105 \mathrm{SD}$ female rats were used to establish the rat with dorsal spinal cord injury model (modified Allen's method). The rats weighed from 200 to $250 \mathrm{~g}$ and were provided by the Experimental Animal Center of Chongqing Medical University. They were randomly divided into five treatment groups: saline (NS group), methylprednisolone (MP group), and musk ketone groups (MO1, MO2, and MO3 groups). The Swash plate test and BBB behavioral score were used to determine neurological function recovery after spinal cord injury. Hematoxylineosin (HE) staining was used to detect general structural changes in spinal cord tissue. The enzyme-linked immunosorbent assay was used for the determination of interleukin 10 (IL-10) in spinal cord tissue. We found that compared with the NS control group, critical angle, BBB score and IL-10 levels in rat spinal cord tissue significantly increased in the MP group and MO groups 7 and 14 days after the operation. HE staining showed that in the NS group, there was hemorrhage, edema, necrosis, axonal demyelination, inflammatory cell infiltration
\end{abstract}


and glial cell response in spinal cord tissue. After 7 days, spinal cord edema and inflammation were reduced and neuronal degeneration and necrosis were not evident in the MP and MO groups. We conclude that musk ketone can reduce secondary damage after spinal cord injury and promote nerve recovery in rats.

Key words: Spinal cord injury; Musk ketone; Nerve function; Interleukin-10

\section{INTRODUCTION}

Spinal cord injury (SCI) is one of the serious diseases commonly seen in the clinic, and it can be divided into primary and secondary injury (Mahabaleshwarkar and Khanna, 2014). Studies have reported that spinal cord injury involves a variety of pathophysiological processes, such as lipid peroxidation, release of excitatory amino acids, inflammation, intracellular calcium overload, microcirculation disturbance, and apoptosis, and the immune inflammatory response is one of the most important pathological processes in secondary damage (Dinc et al., 2013; Ordonez et al., 2013; Smith et al., 2013; Kurtoglu et al., 2014). Inflammatory cytokines are the basic regulatory molecules in the immune response, where they are involved in the regulation of inflammatory reactions in SCI (Xiyang et al., 2014). The necessary inflammatory response is beneficial for functional recovery of SCI, because it can remove tissue debris, induce the secretion of some neurotrophic factors, and promote the recovery of motor function. But after SCI, neuronal necrosis, edema, and blood-spinal cord barrier damage are closely associated with the inflammatory response. After injury, myelin debris can also cause adverse inflammatory response, with nerve regeneration being inhibited (David et al., 2012). Therefore, excessive inflammatory response is still one of the main causes of spinal cord injury. How to adjust the inflammatory response after SCI has become the focus of research in this area. Musk ketone is a ketone first isolated from a natural musk at the beginning of this century. It has a special flavor and is one of the main active ingredients of natural musk (Lovell and Sanders, 1988; Gardeazábal et al., 1992; Hawkins et al., 2002). Systemic administration of musk ketone in mice showed that it can reach various parts of the central nervous system through the blood-brain barrier within 5 min after intragastric administration and within 2 min after intravenous injection (Api and Gudi, 2000). LehmanMcKeeman et al. (1999) showed that musk ketone had multiple pharmacological effects such as promotion of neuronal damage restoration, protection of the central nervous system and prevention of endothelial injury. In addition, studies have shown that musk ketone has significant anti-inflammatory effects (Stuard et al., 1997; Lehman-McKeeman et al., 1999), but the influence of musk ketone on nerve injury recovery after spinal cord injury is unclear. Therefore, the present study focused on the impact of musk ketone on neurological recovery after SCI.

\section{MATERIAL AND METHODS}

\section{Animal grouping and administration}

A total of 105 healthy adult male SD rats were included. They were provided by the 
Experimental Animal Center of Chongqing Medical University, and had a body weight of 200 - $250 \mathrm{~g}$. They were randomly divided into the following treatment groups: normal saline (NS group, $\mathrm{N}=30$ ), methylprednisolone (MP group, $\mathrm{N}=30$ ), and intravenous injection of musk ketone (MO1, MO2, and MO3 groups, 15 in each group).

\section{Methods}

Establishment of pre-condition: Chloral hydrate $(4 \%, 0.35 \mathrm{~mL} / \mathrm{kg})$ was intravenously injected in the tail for anesthesia in all animals. The rats were then fixed in the prone position, and lower back was shaved. The routine disinfected towels were prepared, with the T8 spinous process in the center. A middle back incision was made, the spinous process and lamina were exposed, and T7-10 were removed. For spinous process and laminectomy, the spinal cord was taken as the center, the circular area with a diameter of $5 \mathrm{~mm}$ was revealed. The modified Allen's method was used to hit the dorsal spinal cord. The hammer with a mass of $10 \mathrm{~g}$ was allowed to fall freely on the spinal cord from a height of $2.5 \mathrm{~cm}$. It struck vertically the soft plastic sheet, where one end of the plastic film was concave, which was in direct contact with the endorhachis, allowing the potential energy to pass to the T8 spinal cord.

\section{Sign of successful strike}

Spastic tail swing was noticed, and there was a retraction of the lower limbs and torsolike flutter. Both lower extremities showed flaccid paralysis. The dura, erector spinae, subcutaneous tissue and skin were sutured layer by layer. Intramuscular penicillin at $1.0 \times 10^{5} \mathrm{IU} /$ day was injected in each rat after surgery, and the animals were kept at room temperature of $20-25^{\circ} \mathrm{C}$. Artificial bladder squeeze was performed twice a day until the return of spontaneous urination.

\section{Treatment}

In the MO1, MO2, and MO3 groups, immediately after the operation, 2.5, 5, and 10 $\mathrm{mg} / \mathrm{kg}$ musk ketone were injected in the tail vein, respectively. At 3 and $8 \mathrm{~h}$ after the operation, 5,10 , and $20 \mathrm{mg} / \mathrm{kg}$ musk ketone were injected in the tail vein, respectively. The dose was changed back respectively to $2.5,5$, and $10 \mathrm{mg} / \mathrm{kg}$, given daily for 7 days. In the MP control group, a tail injection of $30 \mathrm{mg} / \mathrm{kg}$ methylprednisolone was performed immediately after the operation and repeated the next day. The NS group was only spinal cord injury with tail vein injection of saline.

\section{Neurological function evaluation after spinal cord injury}

At 1, 3, 7, and 14 days, the oblique plate test was performed and BBB behavioral score determined in rats of each group. The motor function recovery in spinal cord in animals was observed.

\section{Specimen collection}

Six rats of each group were randomly selected 1, 3, 7, and 14 days after the operation. After intraperitoneal anesthesia, the rats were fixed in a supine position, the chest cut open 
and heart exposed under direct vision. A syringe was inserted through the left ventricle into the ascending aorta, and the auricula dextra was cut open. The rats were rapidly perfused with $200 \mathrm{~mL}$ saline containing heparin $(10 \mathrm{U} / \mathrm{mL})$ until the fluid was clear. Next, $200 \mathrm{~mL} 2 \%$ glutaraldehyde was slowly infused at $4^{\circ} \mathrm{C}$ until the limbs and tail hardened. Through the original incision, T8 spinal cord tissue sections were completely removed according to the anatomy, and they were fixed in $4 \%$ paraformaldehyde for $24-48 \mathrm{~h}$.

\section{Pathological morphology of spinal tissues}

Spinal cord tissue was subjected to conventional dehydration, and xylene was used for clearance. The tissue was then embedded in paraffin and sliced. At each time point, three randomly selected sections were stained with HE and observed under the microscope in each rat.

\section{IL-10 detection in spinal cord tissues}

ELISA was used to determine interleukin 10 (IL-10) in spinal cord tissues of rats in each group at different time points. The specific assay was performed strictly according to kit instructions.

\section{Statistical analysis}

The SPSS 13.0 statistical software was used for analysis, and data are reported as means \pm standard deviation (s). ANOVA was used to compare the difference between groups. The pairwise means were compared using the LSD method, $\mathrm{P}<0.05$ indicated a significant difference.

\section{RESULTS}

\section{Evaluation of neurological function after spinal cord injury}

Swash plate test results are shown in Table 1. Compared with the NS group, the critical angle significantly increased in MP treatment group and MO treatment group 7 and 14 days after the operation. BBB behavioral score results were in Table 2. Compared with the NS control group, BBB behavioral score significantly increased in MP and MO treatment groups.

Table 1. Critical angle of swash plate test in each group at different time points.

\begin{tabular}{lcccc}
\hline Groups & $24 \mathrm{~h}$ after surgery & 3 days after surgery & 7 days after surgery & 14 days after surgery \\
\hline NS control & $24.5 \pm 0.43$ & $28.5 \pm 0.55$ & $37.3 \pm 0.56$ & $44.5 \pm 0.49$ \\
MP treatment group & $26.1 \pm 0.65$ & $30.4 \pm 0.57$ & $48.1 \pm 0.63^{*}$ & $49.6 \pm 0.66^{*}$ \\
MO treatment group & $26.9 \pm 0.53$ & $31.6 \pm 0.59$ & $48.0 \pm 0.59^{*}$ & $49.4 \pm 0.51^{*}$ \\
\hline
\end{tabular}

Compared to NS control group, $* \mathrm{P}<0.05$.

Table 2. BBB Behavioral scores in each group at different time points.

\begin{tabular}{lcccc} 
Groups & $24 \mathrm{~h}$ after surgery & 3 days after surgery & 7 days after surgery & 14 days after surgery \\
\hline NS control & $2.35 \pm 0.33$ & $4.53 \pm 0.35$ & $6.23 \pm 0.28$ & $8.56 \pm 0.29$ \\
MP treatment group & $3.16 \pm 0.31$ & $4.74 \pm 0.27$ & $9.41 \pm 0.24^{*}$ & $11.56 \pm 0.36^{*}$ \\
MO treatment group & $3.42 \pm 0.38$ & $5.06 \pm 0.21$ & $9.07 \pm 0.19^{*}$ & $11.47 \pm 0.41^{*}$ \\
\hline
\end{tabular}

Compared to NS control group, $* \mathrm{P}<0.05$. 


\section{Pathological morphology of spinal cord}

For the saline control group, hemorrhage, edema, necrosis, axonal demyelination, inflammatory cell infiltration and glial cell response were seen in spinal cord tissue.

After 7 days, in the MP and MO treatment groups, spinal cord edema and inflammation were reduced, and neuronal degeneration and necrosis were not evident (Figure 1).



Figure 1. Histological results of each groups at 1 day and 7 days after surgery.

\section{IL-10 levels change in spinal cord tissue}

The results are shown in Table 3. Compared with the control group, the MO and MP groups showed at different time points after surgery significantly increased IL-10 levels in the spinal cord tissue of each group $(\mathrm{P}<0.01)$.

Table 3. IL-10 levels in each group at different time points.

\begin{tabular}{lcccc}
\hline Groups & 24 h after surgery & 3 days after surgery & 7 days after surgery & 14 days after surgery \\
\hline NS control & $14.11 \pm 1.43$ & $18.23 \pm 1.36$ & $16.20 \pm 1.38$ & $15.31 \pm 1.27$ \\
MP treatment group & $31.63 \pm 1.35^{*}$ & $38.24 \pm 1.29^{*}$ & $36.42 \pm 1.74^{*}$ & $34.53 \pm 1.06^{*}$ \\
MO treatment group & $30.32 \pm 1.31^{*}$ & $35.06 \pm 1.288$ & $32.47 \pm 1.19^{*}$ & $32.10 \pm 1.31^{*}$ \\
\hline
\end{tabular}

Compared to NS control group, $* \mathrm{P}<0.05$.

\section{DISCUSSION}

Spinal cord injury is divided into primary and secondary injury. Due to the irreversibility of primary injury, treatment mainly focuses on secondary spinal cord injury (Umebayashi et al., 2014). Nowadays, the following factors are considered to be involved in secondary spinal cord injury: ischemia and reperfusion injury, blood - spinal cord barrier damage, free radical generation, neuropeptides, monoamines, intracellular ion disorder, nitric oxide, endothelin, 
vascular endothelial growth factor, platelet activating factor, excitatory amino acids, lipid peroxidation and cell apoptosis (Fong and Hauptman, 2012). Secondary spinal cord injury is the deteriorating process of the mutual cascades of neural biochemical mechanisms and vascular mechanisms. A variety of inflammatory cytokines induce neuronal apoptosis, and their participation in secondary injury is an important factor in recovery of nerve function (Lu et al., 2000).

IL-10 is an important immunoregulatory cytokine, where it has a wide range of biological activity including immunosuppressive, anti-inflammatory and immunomodulatory properties (Baca et al., 2014). Musk ketone significantly inhibits inflammation. Musk ketone can inhibit croton oil-induced granulation cyst fluid exudation. It also has a significant role in reducing inflammatory mediators of cervical degeneration. However, studies have not reported the effect of musk on nerve recovery after spinal cord injury. The results of this study showed that in the MO groups compared with the saline control group, the critical angle, BBB scores, and IL-10 levels in spinal cord tissue significantly increased after 7 and 14 days. The result indicates that musk ketone can reduce secondary damage after spinal cord injury, and thus, it has effects of nerve recovery after spinal cord injury in rats.

\section{REFERENCES}

Api AM and Gudi R (2000). An in vivo mouse micronucleus assay on musk ketone. Mutat. Res. 464: 263-267.

Baca JC, Filippi C, Sachithanantham S, Rodriguez-Calvo T, et al. (2014). Direct infection of dendritic cells during chronic viral infection suppresses antiviral T cell proliferation and induces IL-10 expression in CD4 T cells. PLoS One 9: e90855.

David S, Lopez-Vales R and Wee YV (2012). Harmful and beneficial effects of inflammation after spinal cord injury: potential therapeutic implications. Handb. Clin. Neurol. 109: 485-502.

Dinc C, Iplikcioglu AC, Atabey C, Eroglu A, et al. (2013). Comparison of deferoxamine and methylprednisolone: protective effect of pharmacological agents on lipid peroxidation in spinal cord injury in rats. Spine 38: E1649-E1655.

Fong BM and Hauptman JS (2012). From the bench to bedside: Secondary spinal cord injury, ischemic penumbra after stroke, neural regulation of appetite, microglia in Rett syndrome, signaling pathways in peripheral nerve regeneration. Surg. Neurol. Int. 3: 56.

Gardeazábal J, Arregui MA, Gil N, Landa N, et al. (1992). Successful treatment of musk ketone-induced chronic actinic dermatitis with cyclosporine and PUVA. J. Am. Acad. Dermatol. 27: 838-842.

Hawkins DR, Elsom LF, Kirkpatrick D, Ford RA, et al. (2002). Dermal absorption and disposition of musk ambrette, musk ketone and musk xylene in human subjects. Toxicol. Lett. 131: 147-151.

Kurtoglu T, Basoglu H, Ozkisacik EA, Cetin NK, et al. (2014). Effects of cilostazol on oxidative stress, systemic cytokine release, and spinal cord injury in a rat model of transient aortic occlusion. Ann. Vasc. Surg. 28: 479-488.

Lehman-McKeeman LD, Caudill D, Vassallo JD, Pearce RE, et al. (1999). Effects of musk xylene and musk ketone on rat hepatic cytochrome P450 enzymes. Toxicol. Lett. 111: 105-115.

Lovell WW and Sanders DJ (1988). Photoallergic potential in the guinea-pig of the nitromusk perfume ingredients musk ambrette, musk moskene, musk xylene, musk ketone, and musk tibetene. Int. J. Cosmet. Sci. 10: 271-279.

Lu J, Ashwell KW and Waite P (2000). Advances in secondary spinal cord injury: role of apoptosis. Spine 25: 1859-1866.

Mahabaleshwarkar R and Khanna R (2014). National hospitalization burden associated with spinal cord injuries in the United States. Spinal Cord. 52: 139-144.

Ordonez FJ, Rosety MA, Camacho A, Rosety I, et al. (2013). Arm-cranking exercise reduced oxidative damage in adults with chronic spinal cord injury. Arch. Phys. Med. Rehabil. 94: 2336-2341.

Smith JA, Park S, Krause JS and Banik NL (2013). Oxidative stress, DNA damage, and the telomeric complex as therapeutic targets in acute neurodegeneration. Neurochem. Int. 62: 764-775.

Stuard SB, Caudill D and Lehman-McKeeman LD (1997). Characterization of the effects of musk ketone on mouse hepatic cytochrome P450 enzymes. Fundam. Appl. Toxicol. 40: 264-271.

Umebayashi D, Natsume A, Takeuchi H, Hara M, et al. (2014). Blockade of gap junction hemichannel protects secondary spinal cord injury from activated microglia-mediated glutamate exitoneurotoxicity. J. Neurotrauma 31: 1967-1974.

Xiyang YB, Lu BT, Ya Z, Yuan Z, et al. (2014). Expressional difference, distributions of TGF-beta1 in TGF-beta1 knock down transgenic mouse, and its possible roles in injured spinal cord. Exp. Biol. Med. 239: 320-329. 